\title{
FINGERPRINT CLASSIFICATION BASED ON ORIENTATION FIELD
}

\author{
Zahraa Hadi Khazaal and Safaa S. Mahdi \\ Department of Computer Engineering, Al Nahrain University, Baghdad, Iraq
}

\begin{abstract}
This paper introduces an effective method of fingerprint classification based on discriminative feature gathering from orientation field. A nonlinear support vector machines (SVMs) is adopted for the classification. The orientation field is estimated through a pixel-Wise gradient descent method and the percentage of directional block classes is estimated. These percentages are classified into four-dimensional vector considered as a good feature that can be combined with an accurate singular point to classify the fingerprint into one of five classes. This method shows high classification accuracy relative to other spatial domain classifiers.
\end{abstract}

\section{KEYWORDS}

Orientation Field, Singular point, SVMs Classifier, Feature Vector.

\section{INTRODUCTION}

The maturing of fingerprint identification system and the fingerprint database is expanded therefore, the identification become a problem due to a comparison of the input image with a large fingerprint images taken a long time especially in real time application, so that the classification becomes a vital role in increasing speed and accuracy of the system[1]. By reducing the size of the database through dividing it into classes. SVMs classifier provides elegant solution to different problems such as classification, regression problem, face detection, and handwriting recognition. SVM can be considered a conventional classifier due to immediate determination of decision boundary between training data, this leads to maximization of feature space between classes that minimizing generalization error, also the risk of over fitting is less in SVMs. At last its ability to learning with few training examples. There are many applications of fingerprint that increase the privacy of individuals e.g. (deal with money in electronic wallet, and electoral voting). Fingerprint classified into five classes named as Whorl, Left Loop, Arch, Right loop and Tented arch according to henry classification as shown in Figure (1). The distrubion of classes is uneven. The probability of classes were $0.2790 .029,0.317,0.338,0.029$, and 0.037 for the whorl, right loop, left loop, tented arch and arch respectively [2] as approximatly descriped in figure (2). The significant problem in classification is the similrity of structure and shape of images escepicaly in whrol where covering large spread of print for the same charateristic, this problem called large inter-class variation as shown in figure (3). A classification algorithm was divided into 4 types (i) Rule-based method depend on the number, locations of singularity and geometrical shape of ridge line [3]. (ii) Syntactic approach in this method image was described by small groups of directional components in the orientation image then classification depends on some grammars. (iii) Structural approach was based on a rational graph model for describing the feature. (iv) Cognitive method, for example, neural network, fuzzy and support vector machines (SVMs) were relay on feature vector either from singularity region or directional image and processed it to obtain final classification based on a pyramidal architecture[4]. 


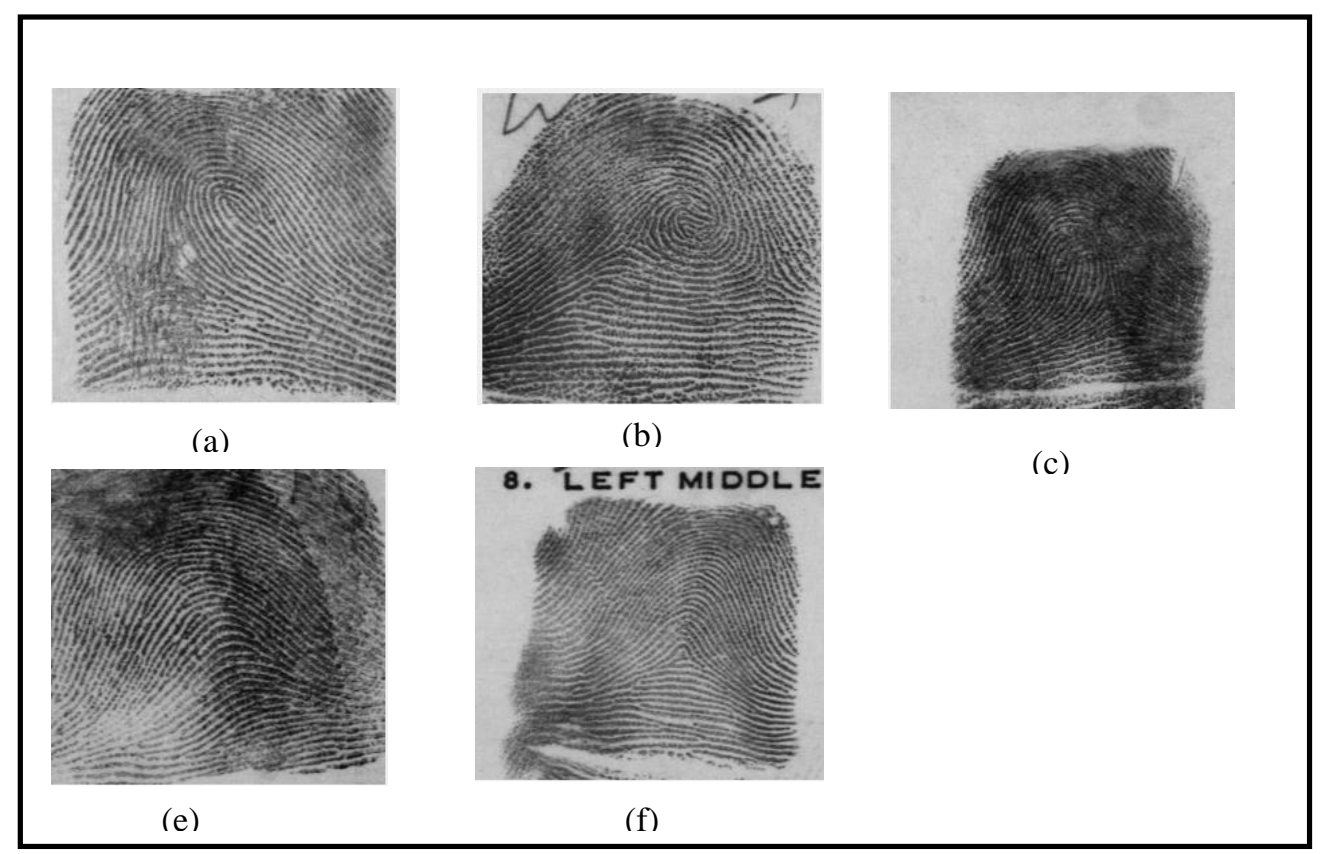

Figure 1. Five samples of a fingerprint from NIST-DB4 database. (a) Right loop, (b)Whorl, (c)Left loop, (e) Arch, (f) Tented arch

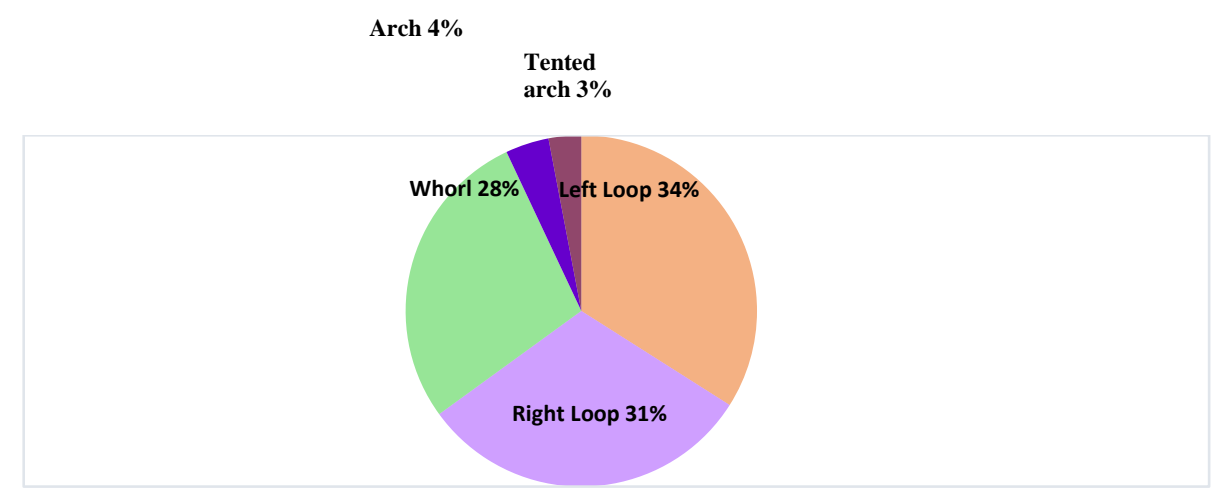

Figure 2. The distribution of henry classification [2].

different classification techniques have been proposed such as hierarchical classifier based on $\mathrm{K}$ Nearest Neighbors (KNN) and SVM for feature extracted from orientation field and complex filter [5] and convolutional Neural Network was used for classification [6] as well as Fuzzy C-Means (FCM) and Naive Bayes classifier demonstrated for classification fingerprint into 4 classes [7] .

In this paper, an efficient method for classification with low time consuming and high accuracy is investigated, uncomplicated but robust against noise based on extracting feature that consists of a directional block percentage of an image and location and number of accurate singular points. We use SVM as a classifier for classification and comparing it with KNN classifier. 
The paper is regulated as follows. Section 2 introduces the proposed method. Section 3, 4, 5 features will be extracted, then section 5 presents classifier that was applied, Section 6 displays the experimental result for two databases, finally conclusions is carried out based on result.
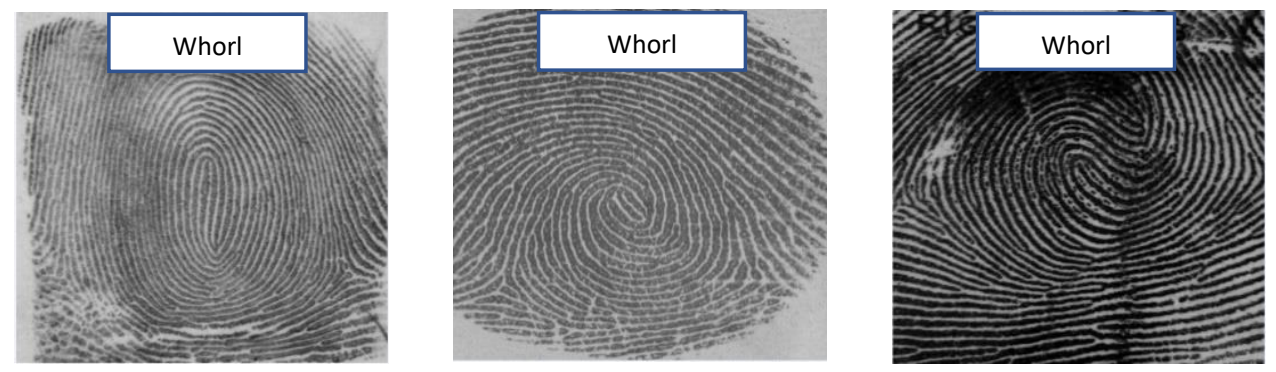

Figure 3. Three whorl images with different characteristics

\section{The Proposed Method}

The basic idea of the proposed method is to extract a sufficient feature to improve time performance and to provide high accuracy for fingerprint image classification in different quality, and that makes recognition of fingerprint to become more easier in large database. Figure (4) demonstrates the block diagram of the proposed method. Support vector machines used for classification.

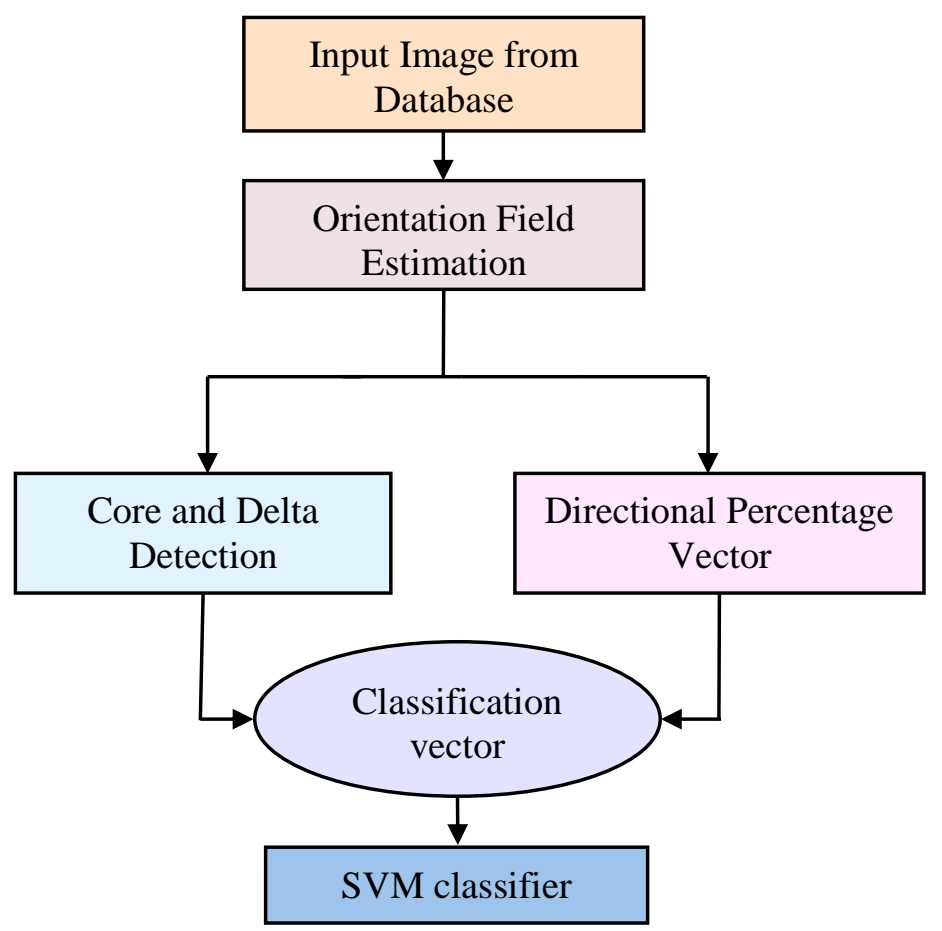

Figure 4. Proposed method block diagram 


\section{Directional Field Estimation by Least Mean Square Algorithm.}

A generic step in the singular point determination is the orientation field that presents the direction and location of the ridge in the fingerprint image. The gradient-based method was used for calculating orientation $\theta$, through different steps[8], these are as follows,

1- Compute the gradient $\partial_{x}(i, j)$ and $\partial_{y}(i, j)$ of each pixel in fingerprint image along the horizontal and vertical direction image based on Sobel Operator that consist of $3 \times 3$ gradient filter as follows: -

$$
\begin{aligned}
& s_{x}=\left[\begin{array}{ccc}
1 & 2 & 1 \\
0 & 0 & 0 \\
-1 & -2 & -1
\end{array}\right] \\
& s_{y}=\left[\begin{array}{lll}
1 & 0 & -1 \\
2 & 0 & -2 \\
1 & 0 & -1
\end{array}\right]
\end{aligned}
$$

2- Calculate the orientation $\theta(i, j)$ of $w \times w$ nonoverlapping blocks centering at pixel $(i, j)$ and can be computed as: -

$$
\begin{aligned}
& V_{x}(\mathrm{i}, \mathrm{j})=\sum_{u=i-\frac{W}{2}}^{i+\frac{W}{2}} \sum_{v=j-\frac{W}{2}}^{j+\frac{W}{2}} 2 G_{x}(\mathrm{u}, \mathrm{v}) G_{y}(u, v) \\
& V_{y}(i, j)=\sum_{u=i-\frac{W}{2}} \sum_{v=j-\frac{W}{2}}^{i+\frac{W}{2}} G_{x}^{2}(u, v)-G_{y}^{2}(u, v)
\end{aligned}
$$

Accordingly,

$$
\theta(i, j)=\frac{1}{2} \tan ^{-1}\left(\frac{V_{x}}{V_{y}}\right)+\frac{\pi}{2}
$$

3- In order to remove broken ridge on the orientation field when gradually varies in the local neighborhood where not genuine singular point appears due to noise, the orientation field is converted to continues vector field to improve accuracy then apply a low pass filter. The continues vector field in the $\mathrm{x}$ and $\mathrm{y}$ components is given by: -

$$
\begin{aligned}
& \phi_{x}(i, j)=\cos (2 \theta(i, j)) \\
& \phi_{y}(i, j)=\sin (2 \theta(i, j))
\end{aligned}
$$

4- Smooth the orientation by low pass filter as follows: -

$$
\phi_{x}^{\prime}(i, j)=\sum_{u=-\frac{w_{\phi}}{2}}^{\frac{w_{\phi}}{2}} \sum_{v=-\frac{w_{\phi}}{2}}^{\frac{w_{\phi}}{2}} G(u, v) . \phi_{x}(i-u w, j-v w)
$$




$$
\phi_{y}^{\prime}(i, j)=\sum_{u=-w_{\phi} / 2}^{w_{\phi} / 2} \sum_{v=-w_{\phi} / 2}^{w_{\phi} / 2} G(u, v) \cdot \phi_{y}(i-u w, j-v w)
$$

5- Estimate the smoothed orientation field centered at pixel $(i, j)$ to obtain a reliable directional field and can be expressed as: -

$$
\theta^{\prime}(i, j) \approx \frac{1}{2} \tan ^{-1} \frac{\phi_{y}^{\prime}(i, j)}{\phi_{x}^{\prime}(i, j)}
$$

Where $\theta^{\prime} \in\{0, \pi / 4, \pi / 2, \pi\}$

6- Depending on Eq (10), calculate the directional block percentage (DBP) for approximation value of $\theta^{\prime}$ for range $0, \pi / 4, \pi / 2$, and $\pi$ by division $N_{d}$ to $N_{T}$ where $N_{d}$ indicates the number of blocks within the $\theta^{\prime}$ over $N_{T}$ that represents the total number of the fingerprint blocks in the orientation field and can be expressed as [9]:-

$$
D P\left(\theta^{\prime}\right)=\frac{N_{d}}{N_{T}} \times 100 \%
$$

After that combine all percentages for all $\theta^{\prime}$ to generate feature vector that will be used later in classification. Figure (5) presents a sample of the orientation field of the fingerprint image.

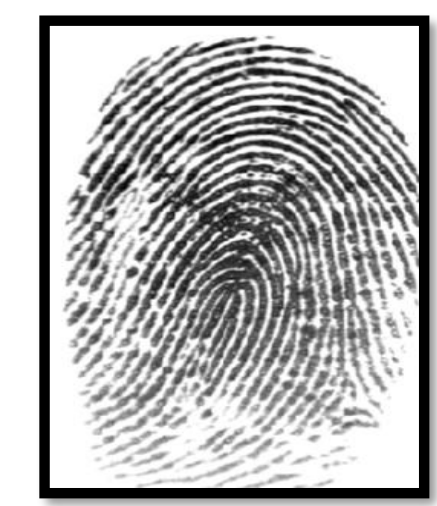

(a)

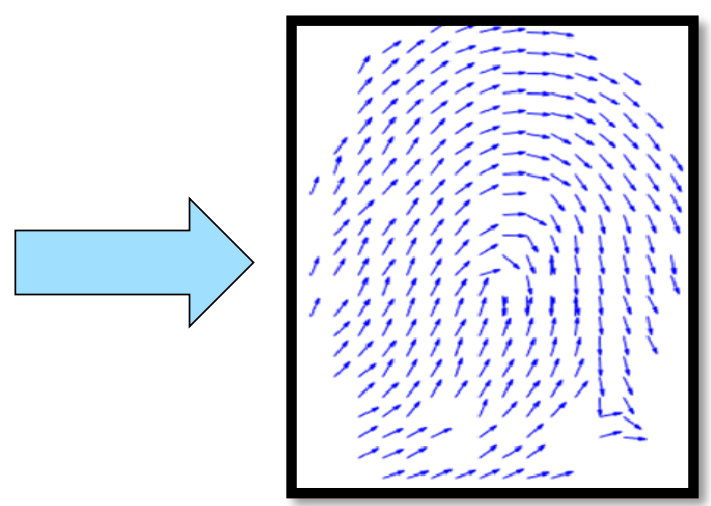

(b)

Figure 5. (a) Original Image (b) Orientation Field

\section{Modified Singular Point Detection}

A Poincare index algorithm is a well-Known method for localizing singular point that includes core and delta point. The Poincare Index (PI) was computed around the closed curved that travels across the counterclockwise route. The value of PI was calculated as the collect of the difference between each two orientation within the curve and can be found at [10]:-

$$
\text { poincare }(i, j)=\frac{1}{2 \pi} \sum_{k=0}^{N-1} \Delta(k)
$$




$$
\Delta(k)=\left\{\begin{array}{lc}
\delta(k) & \text { if }|\delta(k)|<\frac{\pi}{2} \\
\pi+\delta(k) & \text { if } \delta(k) \leq-\frac{\pi}{2} \\
\pi-\delta(k) & \text { otherwise }
\end{array}\right.
$$

Where

$$
\delta(k)=\theta^{\prime}\left(x_{(k+1) \bmod N}, y_{(k+1) \operatorname{modN}}\right)-\theta^{\prime}\left(x_{k}, y_{k}\right)
$$

The PI for core point is 0.5 but for delta is -0.5 , furthermore postprocessing has been used to avoid spurious singular point in the region of scares, crease, blurred prints and other area that affected by noise and to obtain perfect and stable core and delta point, therefore this method should be modified as follows[11]:

1- If the distance between two singular points less than 8 pixels, then all of them should be eliminated.

2- In that case, $\mathrm{N}$ cores or deltas point occurs in the small circular region, then resolve this by averaging cores and deltas.

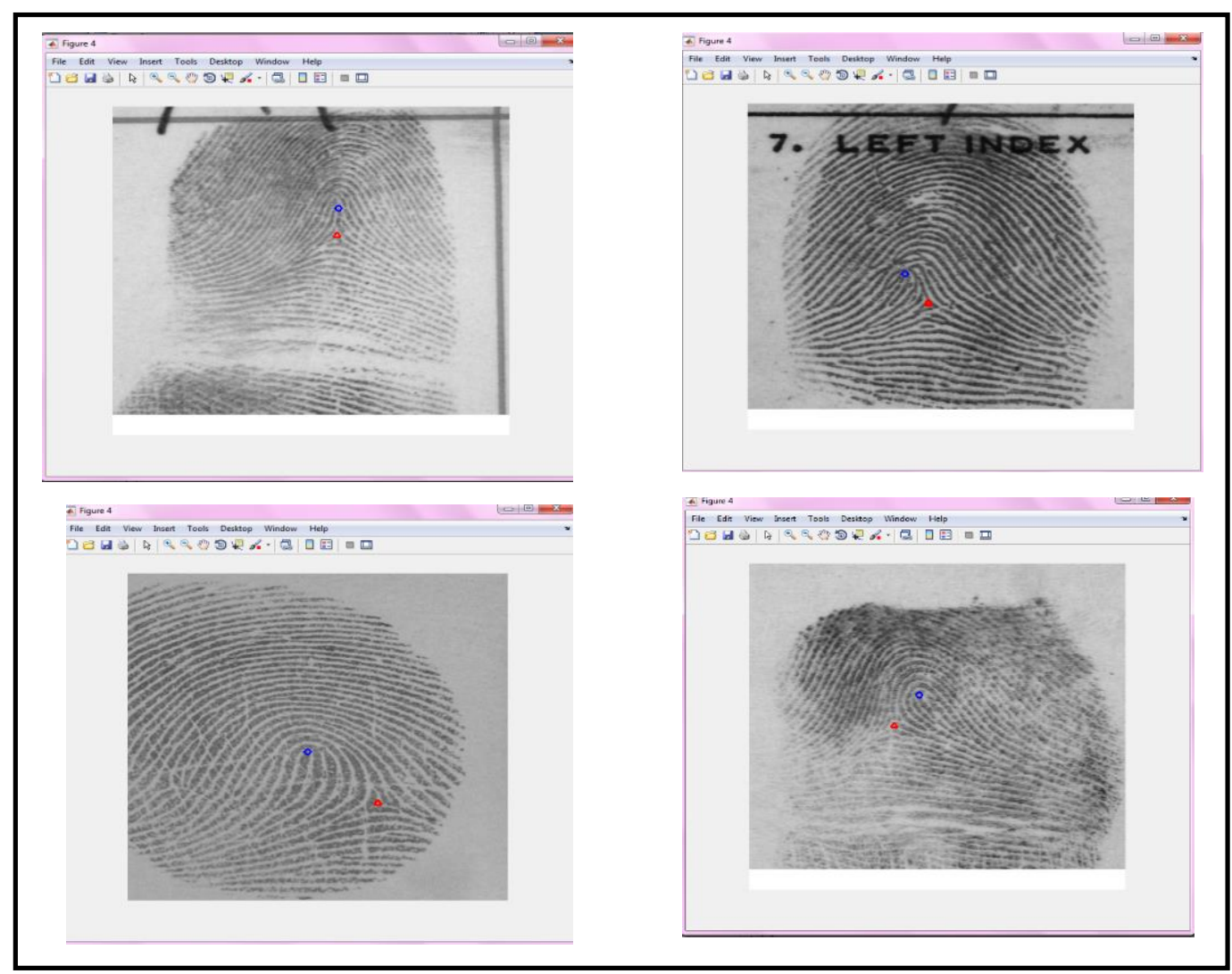

Figure 6. Singular Point for Different Image in NIST SD4 and Second Database

\section{Classification Vector}

The classification vector consists of a directional block percentage for 4 directions, the position, number of core and delta in fingerprint images as given in table 1. 
Table 1. Classification vector

\begin{tabular}{|c|c|c|c|c|c|c|c|c|c|}
\hline \multicolumn{10}{|c|}{ FEATURE VECTOR } \\
\hline $\begin{array}{c}\text { DBP } \\
\text { between } \\
0 \text { and } \\
\pi / 4\end{array}$ & $\begin{array}{c}\text { DBP } \\
\text { between } \\
\pi / \mathbf{4} \text { and } \\
\pi / 2\end{array}$ & $\begin{array}{c}\text { DBP } \\
\text { between } \\
\pi / 2 \text { and } \\
3 \pi / 2\end{array}$ & $\begin{array}{c}\text { DBP } \\
\text { between } \\
3 \pi / 2 \\
\text { and } \pi\end{array}$ & $\begin{array}{c}\text { Core } \\
\mathrm{x} \\
\text { direction }\end{array}$ & $\begin{array}{c}\text { Core } \\
Y \\
\text { direction }\end{array}$ & $\begin{array}{c}\text { Delta } \\
\mathrm{X} \\
\text { direction }\end{array}$ & $\begin{array}{c}\text { Delta } \\
Y \\
\text { Direction }\end{array}$ & $\begin{array}{c}\text { No. } \\
\text { of } \\
\text { cores }\end{array}$ & $\begin{array}{l}\text { No. of } \\
\text { deltas }\end{array}$ \\
\hline
\end{tabular}

\section{SVM CLASSIFIER}

SVM is a supervised training technique that originated from statistical learning theory. SVM performs a good classification via optimal hyperplane determination in infinite dimensional feature space with a maximal margin to the training point that minimizing generalization error. For nonlinearly separable data point a nonlinear function $\phi($.$) which map input features into high$ dimensional feature space $\mathcal{H}$ where the hyperplane classifier applied[12].

For given training data: -

$\mathcal{D}=\left\{\left(x_{i}, y_{i}\right) \mid x_{i} \in \mathbb{R}^{p}, y_{i} \in\{-1,1\}\right\}_{i=1}^{n}$

SVM was trained with learning algorithm from optimization theory Lagrange and gives a sensible load through depending on the inner product in terms of the feature vector in the $\mathcal{H}$ by shaping them in kernel function $\mathrm{K}$, i.e. $\mathrm{K}(\mathrm{x}, \mathrm{y})=\phi(x)^{T} . \phi(y)$. The discriminate function of SVM is given as [13],

$$
g(x)=w^{T} \phi(x)+w_{0}
$$

The SVM determines a saddle point of Lagrange to specify the largest possible margin $L_{D}$ to the nearest training points. The dual form of the Lagrange is $\max L_{D}$ as [13]: -

$$
L_{D}=\frac{1}{2} \sum_{i=1}^{n} \alpha_{i}-\frac{1}{2} \sum_{i=1}^{n} \sum_{j=1}^{n} \alpha_{i} \alpha_{j} y_{i} y_{j} \varphi^{T}\left(x_{i}\right) \varphi\left(x_{j}\right)
$$

Where $y_{i}= \pm 1, i=1, \ldots n$ are class index value

$\alpha_{i}$ Is a Lagrange multiplier that satisfy, subject to the approval of,

$$
\sum_{i=1}^{n} \alpha_{i} y_{i}=0, \quad 0 \leq \alpha_{i} \leq C
$$

Where $\mathrm{C}$ is a regularization parameter that controls fluctuation and training error. Kernel function that is used in a nonlinear SVM must be symmetric function and should satisfy the Mercer's Theorem, thus, one of the important permissible kernel function is a Gaussian kernel as expressed in following equation[14],

$$
K(X, Y)=\exp \left(-\frac{\|X-Y\|}{2 \sigma^{2}}\right)
$$


International Journal of Embedded Systems and Applications (IJESA), Vol 8, No.4,December 2018 Where $\sigma$ is the Gaussian variance, the decision function of SVM described as follows[13],

$$
f(x)=\operatorname{sgn}\left[\sum_{i=1}^{n} \alpha_{i} y_{i} \phi\left(x_{i}\right)^{T} \phi(x)+w_{0}\right]
$$

In criteria of fingerprint classification, we use feature vector that contains 14 dimensions which classify fingerprint using a nonlinear binary classification. To distinguish image first discrimination whorl from dataset that contain (Left loop, Arch, Right Loop, Tented arch) then continuously apply SVM in hierarchal manner to separate the remaining fingerprint images as illustrated in figure (7),

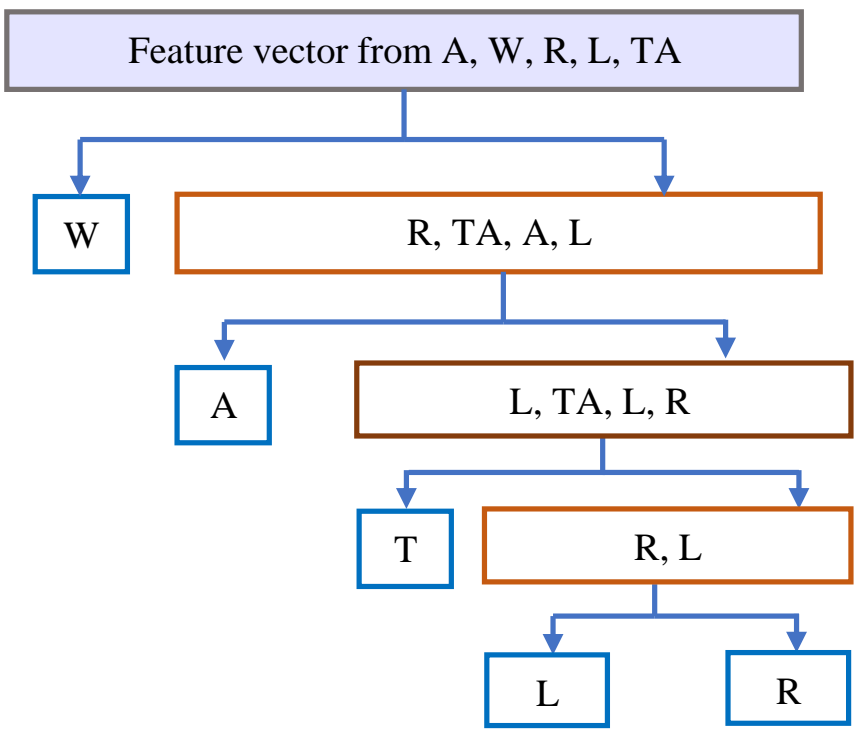

Figure 7. The block Diagram of Five class one VS all SVM Classifier [9]

\section{EXPERIMENTAL RESULTS: -}

In our work, the nonlinear SVMs classifier was applied according to figure (7) in addition, parameters were chosen as $\mathrm{C}=1000, \sigma=0.04$. The performance of the proposed method is presented by conducting on two databases. All program simulated in MATLAB 2017b

\subsection{First DATABASE NIST SPECIAL DATABASE 4 (NIST SD4)}

NIST Special Database 4 (NIST SD4) be composed of 4000 gray scale images with size $512 \times 512$ classified for 5 classes, with 400 images for each class and two images per the same finger, so 500 fingerprints for training and 500 fingerprints for testing were randomly selected, table (2) describes the confusion matrix on NIST SD4. 
International Journal of Embedded Systems and Applications (IJESA), Vol 8, No.4,December 2018 Table (2) Confusion matrix for NIST SD4

\begin{tabular}{|c|c|c|}
\hline \multirow{2}{*}{ Actual Class } & \multicolumn{2}{|c|}{ Predicated Class } \\
\cline { 2 - 3 } & Whorl & Negative \\
\hline Whorl & 96 & 0 \\
\hline Negative & 4 & 400 \\
\hline
\end{tabular}

\begin{tabular}{|c|c|c|}
\hline \multirow{2}{*}{ Actual Class } & \multicolumn{2}{|c|}{ Predicated Class } \\
\cline { 2 - 3 } & Arch & Negative \\
\hline Arch & 94 & 0 \\
\hline Negative & 6 & 300 \\
\hline
\end{tabular}

\begin{tabular}{|c|c|c|}
\hline \multirow{2}{*}{ Actual Class } & \multicolumn{2}{|c|}{ Predicated Class } \\
\cline { 2 - 3 } & Tented Arch & Negative \\
\hline Tented Arch & 100 & 5 \\
\hline Negative & 0 & 195 \\
\hline
\end{tabular}

\begin{tabular}{|c|c|c|}
\hline \multirow{2}{*}{ Actual Class } & \multicolumn{2}{|c|}{ Predicated Class } \\
\cline { 2 - 3 } & Left Loop & Right Loop \\
\hline Left Loop & 100 & 0 \\
\hline Right Loop & 0 & 95 \\
\hline
\end{tabular}

Moreover, the performance evalulatition by using matrices named as precision, sensitivity, specificity, and F-measure which are caluclated from the confucion matrix as follows and it mensioned in figure (8)

$$
\begin{aligned}
& \text { Precision }=\frac{\text { true positive }}{\text { ture positive }+ \text { false positive }} \\
& \text { Rcall }\left(\text { Sensitvity) }=\frac{\text { true positive }}{\text { true positive }+ \text { false negative }}\right. \\
& \text { Specificity }=\frac{\text { true negative }}{\text { true negative }+ \text { false positive }} \\
& F_{-} \text {Measure }=2 *\left(\frac{\text { recall } * \text { precision }}{\text { recall }+ \text { precision }}\right)
\end{aligned}
$$


International Journal of Embedded Systems and Applications (IJESA), Vol 8, No.4,December 2018

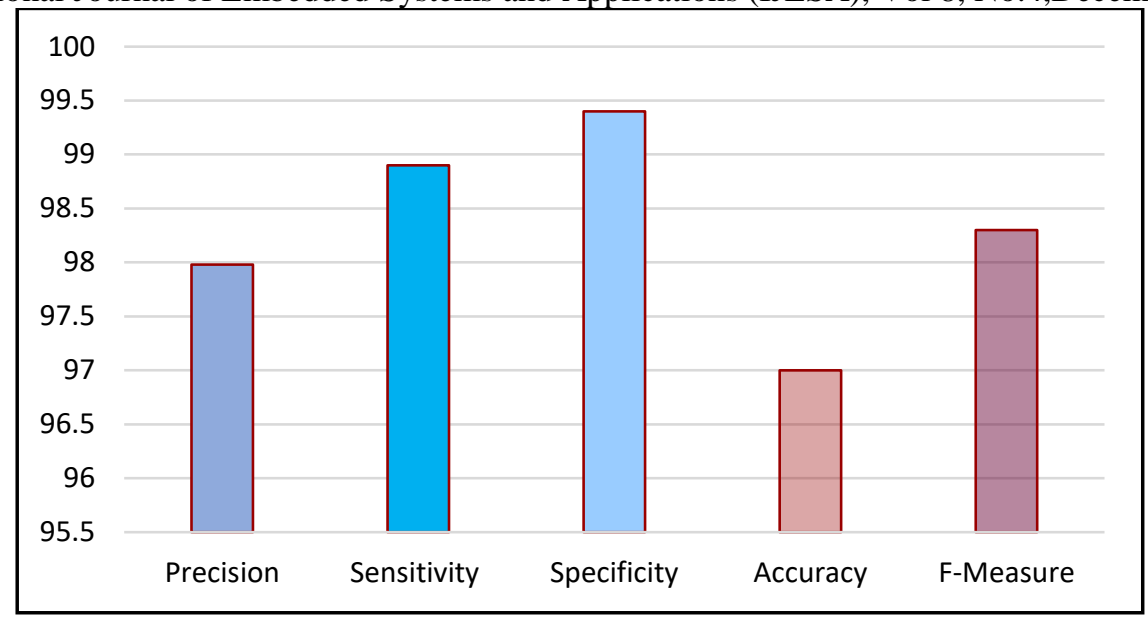

Figure 8. Performance of NIST(SD4)

The result in the table (2) show that SVM allows us to achieve is $94 \%$ accuracy for Whorl, $96 \%$ for Arch, $100 \%$ for Tented Arch and 100\%, 95\% for Left and Right respectively thus total mean accuracy is $97 \%$ and average error percentage is $3 \%$ with average processing time 0.45 second, also figure (8) provides the high ability of a classifier to identify positive and negative label.

\subsection{SECOND DATABASE FOR 100 PERSONS}

we are testing on Second database that contains 1000 fingerprint images for 100 people with size $640 \times 480$, each person has 10 images for the same fingerprint image with different orientation, illumination, and quality half of them were used for test and other half for training. This database was classified into 4 classes as Whorl, Left loop, Right loop, Arch. Table (3), figure (9) shows the confusion matrix for this database and performance measure for database respectively.

Table (3) Confusion matrix for database of 100 persons each one has 10 fingerprint images

\begin{tabular}{|c|c|c|}
\hline \multirow{2}{*}{ Actual Class } & \multicolumn{2}{|c|}{ Predicated Class } \\
\cline { 2 - 3 } & Whorl & Negative \\
\hline Whorl & 177 & 0 \\
\hline Negative & 8 & 315 \\
\hline
\end{tabular}

\begin{tabular}{|c|c|c|}
\hline \multirow{2}{*}{ Actual Class } & \multicolumn{2}{|c|}{ Predicated Class } \\
\cline { 2 - 3 } & Arch & Negative \\
\hline Arch & 45 & 0 \\
\hline Negative & 0 & 270 \\
\hline
\end{tabular}

\begin{tabular}{|c|c|c|}
\hline \multirow{2}{*}{ Actual Class } & \multicolumn{2}{|c|}{ Predicated Class } \\
\cline { 2 - 3 } & Left Loop & Right Loop \\
\hline Left Loop & 103 & 1 \\
\hline Right Loop & 2 & 164 \\
\hline
\end{tabular}




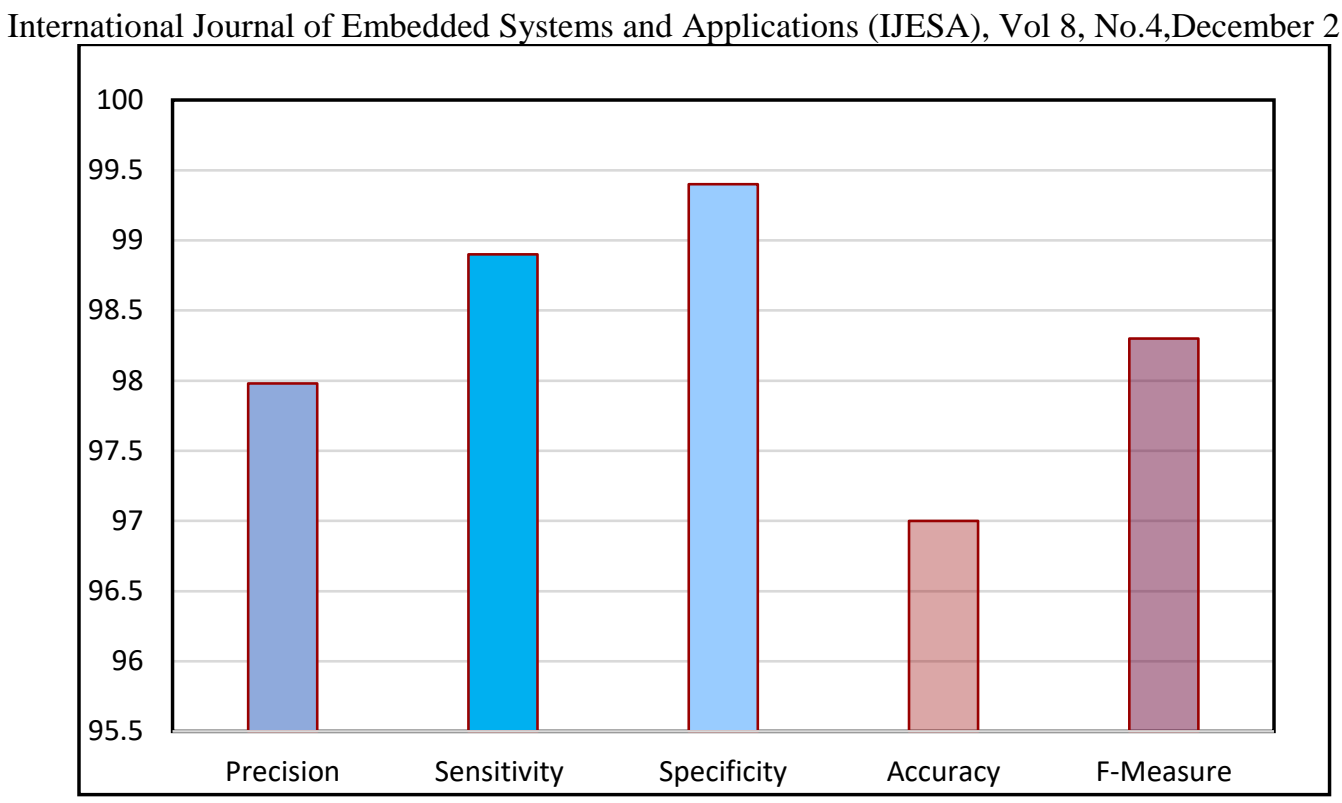

Figure (9) Performance of second database for 100 persons

from table (3) we observe that classifier confusion matrix of a database for 1000 images has given an accuracy of $95.67 \%$ for Whorl and $100 \%, 98 \%, 98.7 \%$ for Arch, Left, and right respectively i.e. the total average accuracy is $97.6 \%$ with average processing time 0.46 second. Also, when we apply KNN hierarchically as SVM classifier, the result of accuracy as described in table (4) and the overall accuracy of both classifiers as shown in figure (10). Thus, KNN do not perform good classification by comparing it with SVM for the same databases because of learning and training phase is very fast which cannot be powerful to noise.

Table 4. Accuracy of KNN classifier

\begin{tabular}{|c|c|c|}
\hline Class & NIST (SD4) for 5 classes & 2 $^{\text {nd }}$ Dataset for 4 Classes \\
\hline Whorl & $88 \%$ & $67 \%$ \\
\hline ARCH & $96 \%$ & $75 \%$ \\
\hline Right Loop & $87 \%$ & $87 \%$ \\
\hline Left Loop & $83 \%$ & $90 \%$ \\
\hline Tented Arch & $57 \%$ & - \\
\hline
\end{tabular}

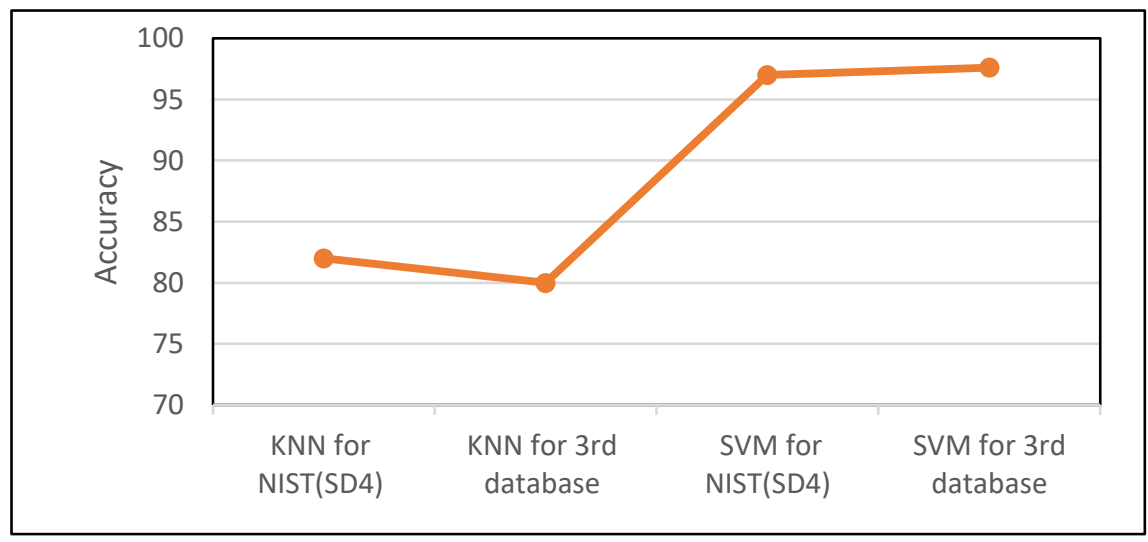

Figure 10. Overall accuracy of KNN and SVM 
International Journal of Embedded Systems and Applications (IJESA), Vol 8, No.4,December 2018 Table (5) show demonstrate comparison to other researchers for different databases and method was used

Table 5. Compares our result with similar works.

\begin{tabular}{|c|c|c|c|c|}
\hline Algorithm & Feature & $\begin{array}{c}5 \text { Class } \\
\text { Accuracy }\end{array}$ & Database & Classifier \\
\hline $\begin{array}{c}\text { Wang, et al } \\
{[15]} \\
2006\end{array}$ & $\begin{array}{c}\text { Directional } \\
\text { image and a } \\
\text { singular point on } \\
\text { ROI image }\end{array}$ & $92.4 \%$ & $\begin{array}{l}\text { NIST-SD4/ } \\
2500 \text { images } \\
1000 \text { train } \\
\text { images, } 1500 \\
\text { test images }\end{array}$ & $\begin{array}{c}\text { Fuzzy Wavelet } \\
\text { Neural network } \\
\text { (FWNN) }\end{array}$ \\
\hline $\begin{array}{l}\text { Ji, Yi [9] } \\
2007\end{array}$ & $\begin{array}{c}\text { Directional } \\
\text { block percentage }\end{array}$ & $96.38 \%$ & $\begin{array}{l}\text { FVC 2004/800 } \\
\text { Images } \\
400 \text { train } \\
\text { images, } 400 \text { test } \\
\text { images }\end{array}$ & SVM \\
\hline $\begin{array}{c}\text { Su Jeon, Yong } \\
\text { [16] } 2017\end{array}$ & - & $97.2 \%$ & $\begin{array}{c}\text { FVC2000, 2002, } \\
2004 \\
788 \text { train } \\
\text { images, } 100 \text { test } \\
\text { images }\end{array}$ & $\mathrm{CNN}$ \\
\hline $\begin{array}{c}\mathrm{Hu}, \text { Xei [17] } \\
2010\end{array}$ & $\begin{array}{c}\text { Primitive } \\
\text { Feature } \\
\text { extracted by a } \\
\text { Genetic } \\
\text { algorithm from } \\
\text { Orientation Field }\end{array}$ & $93.6 \%$ & FVC 2004 & $\begin{array}{c}\text { BP network with } \\
\text { SVM }\end{array}$ \\
\hline $\begin{array}{c}\text { Javed, Usman } \\
\text { [18] } 2011\end{array}$ & Singular point & $95 \%$ & $\begin{array}{l}\text { FVC } 2004 \text { for } \\
320 \text { images }\end{array}$ & $\begin{array}{l}\text { Rule based } \\
\text { method }\end{array}$ \\
\hline Our Proposed & $\begin{array}{c}\text { Directional } \\
\text { percentage and } \\
\text { singular position } \\
\text { and number }\end{array}$ & $\begin{array}{l}97 \%, \\
97.6 \%\end{array}$ & $\begin{array}{l}\text { NIST-SD4 } 1000 \\
\text { images ( } 5 \text { class) } \\
\text { For database of } \\
100 \text { persons, } \\
\text { each one has } 10 \\
\text { images (4 class) }\end{array}$ & Nonlinear SVM \\
\hline
\end{tabular}

In can be seen from table (5) that classification efficiency was improved in four and five classes with less processing time due the hierarchal procedure of classification that gives better result.

\section{Conclusions}

In order to facilitate fingerprint recognition for large databases and reduce required processing time, as well as, increase the efficiency, it is necessary to classify fingerprint first, so that our method gives classification that depends on a robust classification vector which contains the 
International Journal of Embedded Systems and Applications (IJESA), Vol 8, No.4,December 2018 percentage of the directional image and accurate singular point, a nonlinear SVM classifier with RBF kernel and KNN classifier was tested on two Database (NIST-SD4) and another database of 100 people, each one has 10 impressions for the same finger. The experimental result shows that classification accuracy for SVM is $97.6 \%$ for 5 classes in NIST database and $97.6 \%$ for another database for 4 classes while KNN classifier achieve $82 \%$ for NIST $80 \%$ for $2^{\text {nd }}$ database. In summary, the algorithm with SVM classifier that used for fingerprint image classification is more suitable and attractive as well as low computation time through classification for four and five classes due to the hierarchal procedure.

\section{ACKNOWLEDGEMENTS}

The author wishes to thank the mother who gave all tenderness, God's mercy, also would like to thank the Father and sisters who gives safety and strength, may God protect him.

\section{REFERENCES}

[1] R. Wang, C. Han, Y. Wu, and T. Guo, "Fingerprint Classification Based on Depth Neural Network," arXiv, pp. 1-14, 2014.

[2] A. A. Abbood and G. Sulong, "Fingerprint Classification Techniques: A Review," IJCSI Int. J. Comput. Sci. Issues, vol. 11, no. 1, pp. 111-122, 2014.

[3] F. Mirzaei, M. Biglari, and H. Ebrahimpour-komleh, "A Novel Rule-based Fingerprint Classification Approach,” Int. J. Digit. Inf. Wirel. Commun., vol. 3, no. 4, pp. 51-55, 2013.

[4] R. Wang, C. Han, Y. Wu, and T. Guo, "Fingerprint Classification Based on Depth Neural Network," Drug Des. Open Access, vol. 5, no. 2, pp. 1-7, 2014.

[5] K. Cao, L. Pang, J. Liang, and J. Tian, "Fingerprint Classification by a Hierarchical Classifier," Pattern Recognit., vol. 46, no. 12, pp. 3186-3197, 2013.

[6] J. M. Shrein, "Fingerprint Classification Using Convolutional Neural Networks and Ridge Orientation Images," in IEEE Symposium Series on Computational Intelligence (SSCI), 2017, pp. $1-8$.

[7] G. Vitello, F. Sorbello, G. I. M. Migliore, V. Conti, and S. Vitabile, "A Novel Technique for Fingerprint Classification Based on Fuzzy C-Means and Naive Bayes Classifier,” 2014 Eighth Int. Conf. Complex, Intell. Softw. Intensive Syst. A, pp. 155-161, 2014.

[8] G. A. Bahgat, A. H. Khalil, N. S. A. Kader, and S. Mashali, "Fast and Accurate Algorithm for Core Point Detection in Fingerprint Images,” Egypt. Informatics J., vol. 14, no. 1, pp. 15-25, 2013.

[9] L. Ji and Z. Yi, "SVM-based Fingerprint Classification Using Orientation Field," Third Int. Conf. Nat. Comput. IEEE, vol. 2, no. Icnc, pp. 724-727, 2007.

[10] D. Weng, Y. Y. Ã, and D. Yang, "Singular Points Detection based on Multi-resolution in Fingerprint Images,” Neurocomputing, vol. 74, no. 17, pp. 3376-3388, 2011.

[11] G. B. Iwasokun and O. C. Akinyokun, "Fingerprint Singular Point Detection Based on Modififed Poincare Index Method," vol. 7, no. 5, pp. 259-272, 2014.

[12] L. X. W. L. C.-C. J. Kuo, Visual Quality Assessment by Machine Learning. Springer, 2015.

[13] A. R. W. K. D. Copsey, Statistical Pattern Recognition, 3rd Editio. Wiley, 2011. 
International Journal of Embedded Systems and Applications (IJESA), Vol 8, No.4,December 2018

[14] P. Wankhede and D. Doye, "Support Vector Machines for Fingerprint Classification," no. 4, pp. 15.

[15] W. Wang, J. Li, and W. Chen, "Fingerprint Classification Using Improved Directional Field and Fuzzy Wavelet Neural Network,” in IEEE, 2006, vol. 2, pp. 9961-9964.

[16] W. Jeon and S. Rhee, "Fingerprint Pattern Classification Using Convolution Neural Network," Int. J. Fuzzy Log. Intell. Syst., vol. 17, no. 3, pp. 170-176, 2017.

[17] J. Hu and M. Xie, "Fingerprint Classification Based on Genetic Programming," IEEE, vol. 6, pp. 193-196, 2010.

[18] S. Javed, "Computerized System for Fingerprint Classification using Singular Points," IEEE, vol. 1, pp. 4577-657, 2011. 